\title{
Coherent metamaterial absorption of two-photon states with $40 \%$ efficiency
}

\author{
Ashley Lyons, ${ }^{1,2, *}$ Dikla Oren,,${ }^{3, *}$ Thomas Roger, ${ }^{2}$ Vassili Savinov, ${ }^{4}$ João Valente, ${ }^{4,5}$ Stefano Vezzoli, ${ }^{2}$ \\ Nikolay I. Zheludev, ${ }^{4}$ Mordechai Segev, ${ }^{3}$ and Daniele Faccio ${ }^{1,2, \dagger}$ \\ ${ }^{1}$ School of Physics and Astronomy, University of Glasgow, Glasgow, G12 8QQ, United Kingdom \\ ${ }^{2}$ School of Engineering and Physical Sciences, Heriot-Watt University, Edinburgh, EH14 4AS, United Kingdom \\ ${ }^{3}$ Physics Department and Solid State Institute, Technion, 32000 Haifa, Israel \\ ${ }^{4}$ Optoelectronics Research Centre \& Centre for Photonic Metamaterials, University of Southampton, \\ Southampton SO17 1BJ, United Kingdom \\ ${ }^{5}$ School of Engineering, University of Glasgow, Glasgow, G12 8QQ, United Kingdom
}

(Received 29 August 2018; published 14 January 2019)

\begin{abstract}
Multiphoton absorption processes have a nonlinear dependence on the amplitude of the incident optical field, i.e., the number of photons. However, multiphoton absorption is generally weak and multiphoton events occur with extremely low probability. Consequently, it is extremely challenging to engineer quantum nonlinear devices that operate at the single photon level and the majority of quantum technologies have to rely on single photon interactions. Here we demonstrate experimentally and theoretically that exploiting coherent absorption of $N=$ 2 NOON states makes it possible to enhance the number of two-photon states that are absorbed by at most a factor of 2 with respect to a linear absorption process. An absorbing metasurface placed inside a Sagnacstyle interferometer into which we inject an $N=2$ NOON state, exhibits two-photon absorption with $40.5 \%$ efficiency, close to the theoretical maximum. This high probability of simultaneous absorption of two photons holds the promise for applications in fields that require multiphoton upconversion but are hindered by high peak intensities.
\end{abstract}

DOI: 10.1103/PhysRevA.99.011801

\section{INTRODUCTION}

The efficiency with which multiple photons can be instantaneously absorbed by a medium typically has a nonlinear dependence on the amplitude of the optical fields and is therefore negligible at the few-photon level. This is, in general, true for all forms of photon-photon interaction in the quantum (few photon) domain when compared, for example, to high power, classical nonlinear optics where a range of multiphoton effects are readily available (multiphoton absorption, saturable absorption, frequency conversion, etc.) and are therefore currently used in a range of devices. So, although schemes to produce deterministic multiphoton states have been proposed [1], in general, the lack of access to quantum multiphoton processes severely inhibits the use of optics for a large number of applications surrounding quantum technologies. For instance, many quantum logic gates require the interaction between two qubits which could be moderated by the absorption of two photons [2]. Multiphoton absorption at the few-photon level could also lead to photon counting methods with a much higher level of certainty than current techniques [3-5] that also require the identification of events where exactly a set number of photons are absorbed. In addition, quantum states of light have been proposed as a method of surpassing the diffraction limit for photolithography, but this is highly reliant on the simultaneous absorption of a specific number of

\footnotetext{
*These authors have contributed equally to this work.

†daniele.faccio@glasgow.ac.uk
}

photons [6,7]. A mechanism to enhance quantum multiphoton processes would therefore be highly desirable.

Here we demonstrate a route to highly efficient quantum multiphoton processes relying on coherent perfect absorption (CPA), a process that exploits the coherent properties of light to achieve complete absorption [8-10]. Studies have recently shown how CPA can be achieved with ultrathin materials, i.e, with a thickness much less than the wavelength of the light. CPA in such two-dimensional (2D) media has been demonstrated not only with continuous wave sources [11] but also with pulsed sources on the femtosecond timescale [12] and has been extended to investigations into the coherent control of nonlinear effects such as four-wave mixing [13]. Although in what follows we will discuss the quantum phenomenon of two-photon absorption in a thin absorber, it is worth noting that the absorber with no intrinsic nonlinearity that interacts with two incident waves and two outgoing waves is a four port device that can provide nonlinear input-output signal dependencies even in the classical regime of wave interactions. Such nonlinear response results from the redistribution of energy among ports without introducing signal distortion and may be used to small-signal amplifier, summator, and invertor functions for optical signals [14]. For instance, at a constant intensity and zero phase difference between input and pump waves, the output light intensity will be a nonlinear function of input light intensity.

Further studies have started to reveal how CPA interacts with quantum states of light and experiments using single photons have shown that deterministic complete absorption or 
complete transmission of the photons can be achieved $[15,16]$. However, very few investigations have addressed the coherent absorption of quantum multiphoton states, with just a handful of examples available in the literature [17-20].

It was shown in a recent proof-of-principle experiment that two-photon states may exhibit the effects of CPA where a weak modulation in the coincidence count rate was observed, with graphene acting as the thin absorbing medium [19]. Two main factors stand out in this work: The weak modulation that is only of order of a few percent and the lack of a protocol that would enable one to quantitatively estimate the efficiency of the two-photon absorption process. The latter issue is due to the inability for any device to directly measure zero-photon states. Overall, the weak modulation in the coincidence counts indicates that the efficiency in this experiment was far from the theoretical limit of $50 \%$, predicted by theoretical studies $[17,18]$ and thus fails to provide evidence that high absorption probabilities are achievable. Naturally, this will continue to limit the possibilities where coherent absorption of quantum states can be employed, for example, for photonic logic gates [14]. Provided that efficiencies on the scale of this 50\% limit can be demonstrated, CPA can provide a pathway to two-photon absorption schemes which are not accessible with classical states of light (that are always limited to a maximum of $25 \%$ ).

In this work we demonstrate that absorption probabilities on the scale of this fundamental $50 \%$ limit are possible. To achieve this, we use a bespoke metamaterial with absorption, reflection, and transmission coefficients close to the optimum values. We also introduce a measurement protocol which quantifies the amount of two-photon absorption from a simple measurement of only the rate of coincidence events between pairs of detectors with no free fitting parameters. By using this model we demonstrate two-photon coherent absorption with $40 \%$ efficiency, the highest ever reported. In fact, the results presented here prove that the theoretical limit on coherent absorption of multiphoton states can be approached and pave the way for using two-photon coherent absorption in various applications ranging from quantum gates to probing quantum coherence in biological molecules.

\section{THEORY}

Our results rely on the use of an important class of multiphoton quantum states, known as NOON states, which have the form $\left|\Psi_{\text {NOON }}\right\rangle=1 / \sqrt{2}\left(\left|N_{a}, 0_{b}\right\rangle+e^{-i N \theta}\left|0_{a}, N_{b}\right\rangle\right.$ ) (where $N$ is the number of photons, $a$ and $b$ represent two possible modes that the photons can occupy and $\theta$ is an arbitrary phase shift). It can be shown that, for maximum efficiency CPA in an infinitely thin film with a linear absorption $\alpha=0.5$, the reflection and transmission coefficients must be equal in magnitude with a relative phase between them that is equal to $0[10,11,14,21,22]$. A good approximation to this scenario can be engineered using metamaterials with thickness, $d \ll \lambda$ where $\lambda$ is the wavelength of the photons. The output states from such a metamaterial beamsplitter with an $N=2$ NOON input state, are summarized in Table I [17]: by varying the NOON phase $\theta$, the output states change from a single-photon state to a mixture of two-photon states and zero-photon states (where both photons are absorbed). The last implies that, for
TABLE I. Output states from a beamsplitter with $50 \%$ loss for the two opposite input NOON phases, $\theta=0$ (indicated as the $\left|2_{+}\right\rangle$ input state) and $\theta=\pi$ (indicated as the $\left|2_{-}\right\rangle$input state). $\left|\psi_{2}\right\rangle=$ $1 / 2\left(\left|1_{a}, 1_{a}\right\rangle+\left|2_{-}\right\rangle\right)$.

\begin{tabular}{lcc}
\hline \hline$r \& t$ Phase & Input & Output \\
\hline 0 & $\left|2_{+}\right\rangle$ & $1 / 2\left(\left|0_{a}, 0_{b}\right\rangle\left\langle 0_{a}, 0_{b}|+| \psi_{2}\right\rangle\left\langle\psi_{2}\right|\right)$ \\
0 & $\left|2_{-}\right\rangle$ & $\mp\left|1_{ \pm}\right\rangle$ \\
\hline \hline
\end{tabular}

the correct phase of the interferometer $(\theta=0,2 \pi, 4 \pi, \ldots)$, one can expect $50 \%$ simultaneous absorption of both photons. Note that the time-averaged number of photons measured per input pair will always be 1, therefore more sophisticated techniques need to be employed to fully demonstrate this effect. In other words, photon counters cannot directly provide the amplitude of the zero-photon state. We show that this can, however, be achieved by measuring the amplitudes of the output two-photon states together with the system losses, and then inferring the zero-photon states from these measurements.

We build a theoretical model which accounts for the three main processes in the experiment: (i) The generation of two correlated single photons via spontaneous parametric down-conversion; (ii) the formation of an $N=2$ NOON state by combining the generated photons onto a lossless beamsplitter; and (iii) the interaction of the $N=2$ NOON state with the lossy metamaterial beamsplitter and the final measurement of the resultant coincident photon counts. In more detail, the input density matrix $\rho_{0}$ evolves according to

$$
\rho=\sum_{i, j, k=0}^{5} F_{k} \mathcal{U}_{2} E_{j}^{p_{2}, q_{2}} \mathcal{U}_{1} E_{i}^{p_{1}, q_{1}} \rho_{0} E_{i}^{p_{1}, q_{1} \dagger} \mathcal{U}_{1}^{\dagger} E_{j}^{p_{2}, q_{2} \dagger} \mathcal{U}_{2}^{\dagger} F_{k}^{\dagger},
$$

where the indices $i, j, k$ indicate the sum over the dimension of the noise Hilbert space (used to describe the trivial and coherent losses, see supplemental information [23]). The input SPDC state is $\rho_{0}=|11\rangle\langle 11|$, where we use the ordered basis to describe states with up to two photons $|00\rangle,|01\rangle,|10\rangle$, $|11\rangle,|20\rangle,|02\rangle . E_{i}, E_{j}$ are the operators accounting for system losses (absorption, reflection from optical components, scattering from imperfections, etc.) between the SPDC crystal and Hong-Ou-Mandel Beamsplitter (HOM BS), and then between the HOM BS and the metamaterial film, respectively. The unitary operator $\mathcal{U}_{1}$ describes the lossless HOM BS, while $\mathcal{U}_{2}$ is the unitary propagation operator describing the phase shift in the interferometer. Finally, $F_{k}$ are the operators describing the lossy metamaterial BS. The parameters in the loss operators $E_{i}$ are derived from the measured transmission of each individual interferometer arm, as described in more detail in the Supplementary Materials [23]. It is then possible to calculate the coefficient of the $|00\rangle$ basis vector from measurements at the metamaterial output, as described below.

\section{EXPERIMENTAL SETUP}

The experimental layout consists of two cascaded interferometers shown in Fig. 1(a) and described in more detail in the Supplementary Materials [23]. The first interferometer, based 


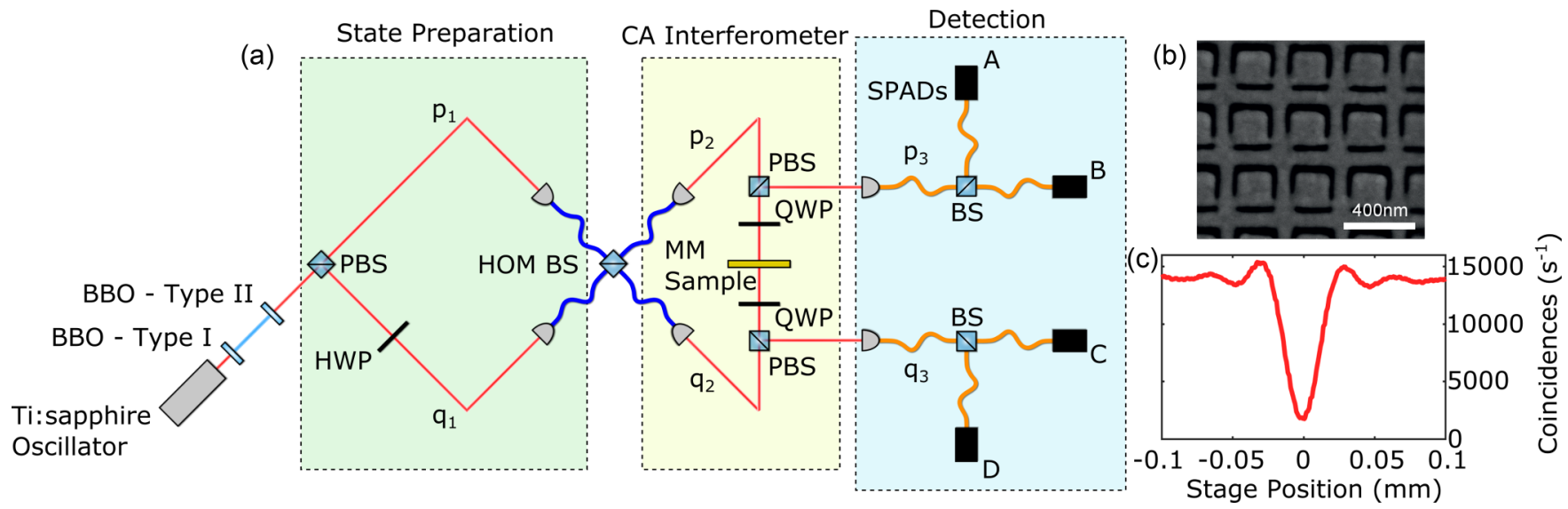

FIG. 1. (a) Schematic of the experiment split into three sections. The "State Preparation" section creates the $N=2$ NOON states using Hong-Ou-Mandel (HOM) interference. The second section ("Coherent Absorption Interferometer") is a modified Sagnac interferometer using circular polarization at the metamaterial sample (MM Sample). Light is coupled out of the "CA Interferometer" by rotating back to linear polarization and using polarizing beamsplitters (PBS) to achieve a high extraction efficiency. Finally, the photons are counted in the "Detection" section which consists of fiber-coupled 50:50 beamsplitters (BS) and four single photon avalanche diodes (SPADs). (b) SEM image of the metamaterial made up of an array of split ring resonators milled into a 50-nm-thick free-standing gold film by a focused ion beam. (c) The HOM dip as measured at the output ports of the HOM BS by translating one of the fiber-couplers at one of the input ports along the beam propagation direction.

on a lossless BS, generates the NOON states and these are sent to the second "coherent absorption" (CA) interferometer. This consists of two counterpropagating arms which meet at the free-standing metamaterial sample [see Fig. 1(b) and supplementary information for the details of the metamaterial film] in the same fashion as a Sagnac interferometer, which is modified to allow for complete coupling out of the interferometer via polarising beamsplitters (PBS) and quarter waveplates (QWP). The use of circular polarization has minimal effect in terms of the measured two-photon state absorption. Both of the output ports are then coupled to multimode fibers and sent via fiber-coupled beamsplitters to four SPAD detectors, which we label $A, B, C$, and $D$. Previous experiments using plasmonic interactions (such as those utilized by our metamaterial) demonstrated that they can be used for quantum interference and entanglement experiments without significant decoherence of the state $[20,24,25]$.

\section{RESULTS}

The total number of two-photon states is determined by measuring the total number of two-fold coincident counts between all possible combinations of detectors $A, B, C, D$. To provide an accurate estimate, there are two considerations which must be taken into account. The first is due to the probabilistic nature of the beamsplitters used for detection which will only separate $50 \%$ of the incident two-photon states. The total number of two-photon states is therefore,

$$
C_{\text {Total }}=2\left[C_{A B}+C_{C D}\right]+\left[C_{A C}+C_{A D}+C_{B C}+C_{B D}\right],
$$

where $C_{i j}$ represents the number of coincident counts between detectors $A, B, C, D$ as indicated in the subscripts $i$ and $j$. The second consideration is the loss throughout the system due to fiber coupling efficiency and non-unity transmission of the components. This is accounted for by the $E_{i}$ matrices in Eq. (1). To recover the output state from measurements after the metamaterial beamsplitter, we model the system by Eq. (1). The experimentally measured coincidence rates are then compared to those predicted by our theoretical model. We do this by renormalizing the rates according to Eq. (2) and by accounting for the measured detector and fibre coupling efficiencies $\left(\eta_{\text {detector }}=0.62\right.$ and $\eta_{\text {coupling }}=0.7$, respectively). Figure 2 shows the total coincidence counts $C_{\text {Total }}$ corresponding to two-photon states (circles) as the phase of the interferometer is varied, and compares this to the

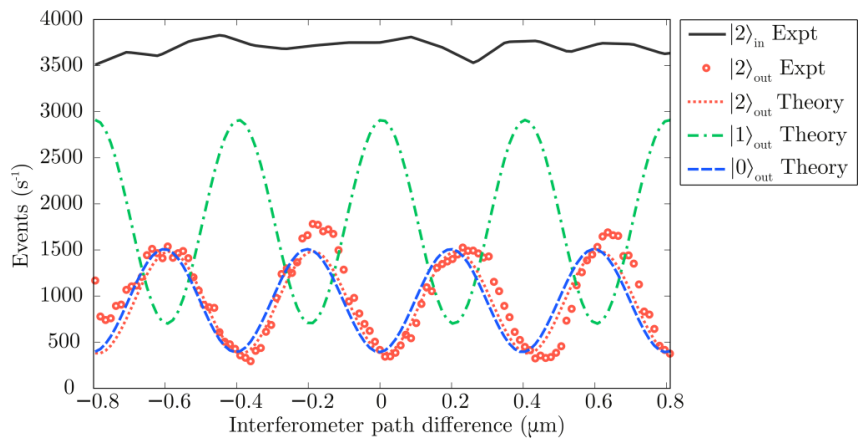

FIG. 2. Amplitudes of zero-, one-, and two-photon states as a function of the interferometer phase. The plots are based on the experimentally measured values of all coincidence-count combinations, incorporating the losses at each stage of the setup. The number of input NOON states is estimated from the total number of coincident counts measured without the absorbing sample and the (separately mmeasured) HOM visibility [Fig. 1(c)]. We note the good agreement between the measured $|2\rangle_{\text {out }}$ and the theoretical estimate of the same quantity, with no free parameters, indicating that the model indeed describes the setting well. 


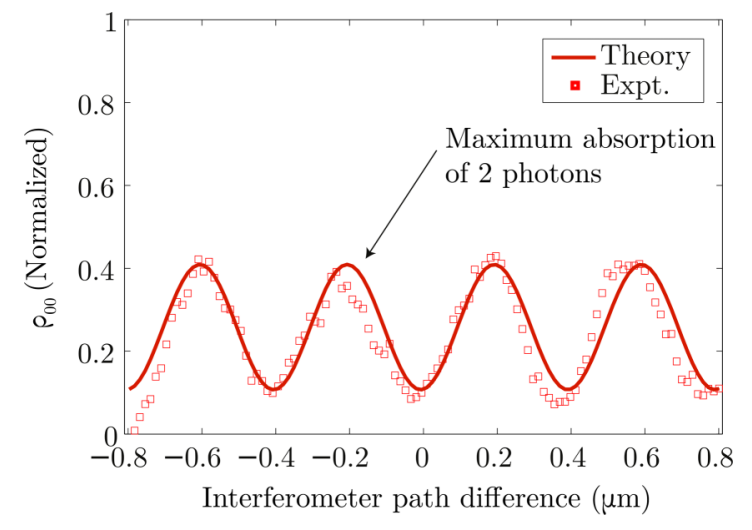

FIG. 3. Variation of the two-photon absorption coefficient $\left(\rho_{00}\right)$ as a function of the interferometer path difference, measured at the metamaterial sample. The squares represent the experimentally measured component, which has been interpreted using the theoretical model including losses. The solid line represents the theoretically calculated $\rho_{00}$ component with no free parameters.

corresponding theoretical estimate (red curve). The good agreement with no free parameters indicates the high fidelity of the model. The amount of two-photon absorption is then quantified directly from the output density matrix Eq. (1) projected onto the basis vector $|00\rangle$. A similar operation can be performed for the one-photon states. These results are plotted in Fig. 2 along with the total two-photon input rate $|2\rangle_{\text {in }}$ measured at the HOM BS output (dashed line). The ratio between $|0\rangle_{\text {out }}$ and $|2\rangle_{\text {in }}$ provides a direct estimate of the total absorption of the two-photon state and is found to reach a maximum of $40.5 \%$ (periodic with the interferometer phase). This approaches the theoretical maximum of $50 \%$ (see supplemental information for more details [23]). This result is shown differently in Fig. 3 where we plot the normalized zerophoton state coefficient $|0\rangle_{\text {out }}=\left(|2\rangle_{\text {in }}-|1\rangle_{\text {out }}-|2\rangle_{\text {out }}\right) /|2\rangle_{\text {in }}$ together with the theoretical prediction (where all quantities in the formula are evaluated from the theoretical model).

Finally, in Fig. 4 we show the expected enhancement of the absorption for a range of NOON states $(N=1$ to $N=7)$ for the parameters of the metamaterial used in the experiments and for the ideal case of $\alpha=0.5$. In the case of the simple $N$ independent-photon absorption the probability of absorbing
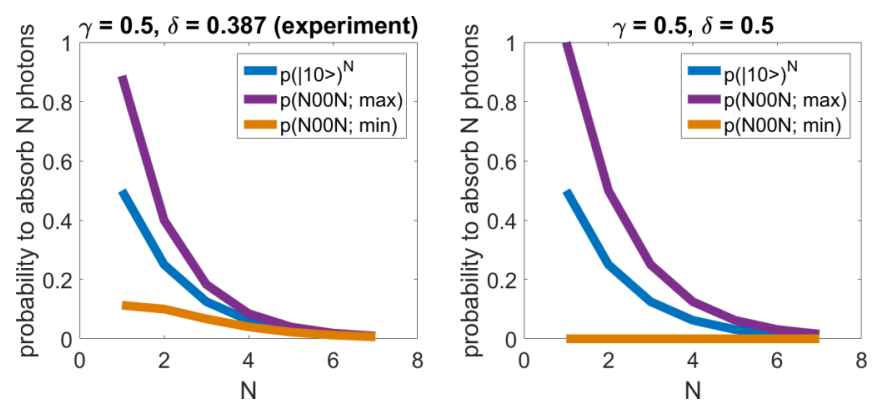

FIG. 4. Enhancement and predicted controllability of the absorption probability of exactly $N$ photons from a NOON state (max: upper purple line, min: lower orange line) compared to $N$ independent photons (center blue line). (a) Parameters for the metasurface used in the experiment. (b) Parameters for maximum enhancement $(\gamma=0.5)$. all $N$ photons scales as the absorption $\gamma$ of one photon to the $N$ th power, i.e., $\mathrm{p}\left(|1,0\rangle^{N}\right)=\gamma^{N}$. On the other hand, for NOON states $p(\mathrm{NOON})=\gamma^{N}+\delta^{N}$ where $\delta=r t^{*}+r^{*} t$. There is therefore an improvement of a maximum factor $2 \times$ using NOON states. The results of this work for $N=2$ can therefore be generalized to higher-order NOON states without loss of generality.

\section{CONCLUSION}

In summary, we provide experimental evidence of efficient coherent two-photon interaction, in the form of two-photon state absorption in a metamaterial. The two-photon absorption rate is quantified through a specifically designed measurement protocol and was recorded to be close to the theoretical maximum of $50 \%$, two times higher than the classical limit and thus manifests the role of entanglement during the perfect absorption process. We note that we only need to measure coincidence rates between the four detectors and characterization of the individual optical components (i.e., losses and transmission), to yield the full density matrix; thereby providing the coefficients of all possible output states composed of zero, one, and two photons. With no free parameters, the model is able to reproduce the total coincidence rates measured in the experiment. This allows us to retrieve the zerophoton amplitudes, even without a method to characterize the one-photon amplitudes directly.

The mechanism of absorption observed here is via conversion to localized plasmons. There have also been a number of recent quantum optics studies that couple photons to traveling plasmons to study their coherence properties. NOON state coherent absorption would be a route to efficient generation of traveling plasmon Fock-states with coherent control over the plasmon number (one or two) by changing the interferometer phase.

Another opportunity could rely on the temporal entanglement that is shared by the signal and idler photons produced by SPDC, which can give rise to an increase in the efficiency with which both photons can be absorbed by systems requiring pumping on an ultrafast timescale yet have very narrowband transitions [26]. The efficiency of such effects could potentially be enhanced to yield an absorption cross section close to unity by combining them with the coherent mechanism demonstrated here. Finally, although a 50\% absorption was specifically tailored with the metamaterial, other surfaces or samples with lower absorption can also be used for coherent absorption by placing these inside a cavity, as in the original proposal for coherent absorption [8], thus providing a route to extending NOON state absorption to any desired material.

All data related to this work may be found at the University of Glasgow [27].

\section{ACKNOWLEDGMENTS}

D.F. acknowledges support from the European Research Council under the European Union's Seventh Framework Programme (FP/2007-2013)/ERC, Grant No. GA 306559, the Engineering and Physical Sciences Research Council (EPSRC, UK, Grants No. EP/M006514/1 and No. EP/M01326X/1) and the Leverhulme Trust. 
[1] C. Reimer, M. Kues, P. Roztocki, B. Wetzel, F. Grazioso, B. E. Little, S. T. Chu, T. Johnston, Y. Bromberg, L. Caspani, D. J. Moss, and R. Morandotti, Generation of multiphoton entangled quantum states by means of integrated frequency combs, Science 351, 1176 (2016).

[2] P. Kok, W. J. Munro, K. Nemoto, T. C. Ralph, J. P. Dowling, and G. J. Milburn, Linear optical quantum computing with photonic qubits, Rev. Mod. Phys. 79, 135 (2007).

[3] N. Lusardi, J. W. N. Los, R. B. M. Gourgues, G. Bulgarini, and A. Geraci, Photon counting with photon number resolution through superconducting nanowires coupled to a multi-channel TDC in FPGA, Rev. Sci. Instrum. 88, 035003 (2017).

[4] A. Divochiy, F. Marsili, D. Bitauld, A. Gaggero, R. Leoni, F. Mattioli, A. Korneev, V. Seleznev, N. Kaurova, O. Minaeva, G. Gol'tsman, K. G. Lagoudakis, M. Benkhaoul, F. Lévy, and A. Fiore, Superconducting nanowire photon-number-resolving detector at telecommunication wavelengths, Nat. Photon. 2, 302 (2008).

[5] E. A. Dauler, A. J. Kerman, B. S. Robinson, J. K. W. Yang, B. Voronov, G. Goltsman, S. A. Hamilton, and K. K. Berggren, Photon-number-resolution with sub-30-ps timing using multielement superconducting nanowire single photon detectors, J. Mod. Opt. 56, 364 (2009).

[6] A. N. Boto, P. Kok, D. S. Abrams, S. L. Braunstein, C. P. Williams, and J. P. Dowling, Quantum Interferometric Lithography: Exploiting Entanglement to Beat the Diffraction Limit, Phys. Rev. Lett. 85, 2733 (2000).

[7] J. P. Dowling, Quantum optical metrology - the lowdown on high-NOON states, Contemp. Phys. 49, 125 (2008).

[8] Y. D. Chong, L. Ge, H. Cao, and A. D. Stone, Coherent Perfect Absorbers: Time-Reversed Lasers, Phys. Rev. Lett. 105, 053901 (2010).

[9] S. Longhi, PT-symmetric laser absorber, Phys. Rev. A 82, 031801(R) (2010).

[10] D. G. Baranov, A. Krasnok, T. Shegai, A. Alù, and Y. Chong, Coherent perfect absorbers: linear control of light with light, Nat. Rev. Mater. 2, 17064 (2017).

[11] J. Zhang, K. F. MacDonald, and N. I. Zheludev, Controlling light-with-light without nonlinearity, Light Sci. Appl. 1, e18 (2012).

[12] S. M. Rao, J. J. F. Heitz, T. Roger, N. Westerberg, and D. Faccio, Coherent control of light interaction with graphene, Opt. Lett. 39, 5345 (2014).

[13] S. M. Rao, A. Lyons, T. Roger, M. Clerici, N. I. Zheludev, and D. Faccio, Geometries for the coherent control of four-wave mixing in graphene multilayers, Sci. Rep. 5, 15399 (2015).

[14] X. Fang, K. F. MacDonald, and N. I. Zheludev, Controlling light with light using coherent metadevices: all-optical transistor, summator and invertor, Light Sci. Appl. 4, e292 (2015).

[15] T. Roger, S. Vezzoli, E. Bolduc, J. Valente, J. J. F. Heitz, J. Jeffers, C. Soci, J. Leach, C. Couteau, N. I. Zheludev, and D. Faccio, Coherent perfect absorption in deeply subwavelength films in the single-photon regime, Nat. Commun. 6, 7031 (2015).

[16] S. Huang and G. S. Agarwal, Coherent perfect absorption of path entangled single photons, Opt. Express 22, 20936 (2014).

[17] J. Jeffers, Interference and the lossless lossy beam splitter, J. Mod. Opt. 47, 1819 (2000).

[18] S. Barnett, J. Jeffers, A. Gatti, and R. Loudon, Quantum optics of lossy beam splitters, Phys. Rev. A 57, 2134 (1998).

[19] T. Roger, S. Restuccia, A. Lyons, D. Giovannini, J. Romero, J. Jeffers, M. Padgett, and D. Faccio, Coherent Absorption of N00N States, Phys. Rev. Lett. 117, 023601 (2016).

[20] B. Vest, M. C. Dheur, É. Devaux, A. Baron, E. Rousseau, J. P. Hugonin, J. J. Greffet, G. Messin, and F. Marquier, Anticoalescence of bosons on a lossy beam splitter, Science 356, 1373 (2017).

[21] S. Dutta-Gupta, O. J. F. Martin, S. D. Gupta, and G. S. Agarwal, Controllable coherent perfect absorption in a composite film, Opt. Express 20, 1330 (2012).

[22] S. Thongrattanasiri, F. H. L. Koppens, and F. Javier García De Abajo, Complete Optical Absorption in Periodically Patterned Graphene, Phys. Rev. Lett. 108, 047401 (2012).

[23] See Supplemental Material at http://link.aps.org/supplemental/ 10.1103/PhysRevA.99.011801 for a detailed description of the experimental layout and metamaterial, characterization of the setup, and a theoretical model describing losses within the system.

[24] J. S. Fakonas, H. Lee, Y. A. Kelaita, and H. A. Atwater, Twoplasmon quantum interference, Nat. Photon. 8, 317 (2014).

[25] J. S. Fakonas, A. Mitskovets, and H. A. Atwater, Path entanglement of surface plasmons, New J. Phys. 17, 023002 (2015).

[26] F. Schlawin, K. E. Dorfman, B. P. Fingerhut, and S. Mukamel, Suppression of population transport and control of exciton distributions by entangled photons, Nat. Commun. 4, 1782 (2013).

[27] A. Lyons, D. Oren, T. Roger, V. Savinov, J. Valente, S. Vezzoli, N. I. Zheludev, M. Segev, and D. Faccio, Coherent metamaterial absorption of two-photon states with $40 \%$ efficiency, http://dx. doi.org/10.5525/gla.researchdata.721 (2019). 